\title{
On the UWB WBAN Radio Channel Research
}

\author{
Matti Hämäläinen $^{*(1)}$, Mariella Särestöniemi ${ }^{(1)}$, Timo Kumpuniemi ${ }^{(1)}$, Jari Iinatti ${ }^{(1)}$, Ryuji Kohno $^{(2)}$ \\ (1) Centre for Wireless Communications, University of Oulu, Finland \\ https://www.oulu.fi/cwc/ \\ (2) Yokohama National University, Yokohama, Japan \\ http://www.kohnolab.dnj.ynu.ac.jp/index-e.html
}

\begin{abstract}
In this paper, radio signal propagation characteristics for ultra wideband (UWB) signal is discussed in a wireless body area network (WBAN) context. The studied system approach considers on-body communications, which, however, is affected also by the in-body parts. Multipath signal components could propagate both on-body and inbody. Depending on the sensor node installations and the tissue types and volumes in the vicinity of them, radio signals propagate in the environment differently. Thus, tissues, and especially highly conductive implants underneath the skin could also contribute to on-body propagation characteristics. The following discussion is based on the research carried out at University of Oulu, Finland.
\end{abstract}

\section{Introduction}

Due to the various benefits the use of wireless body area networks (WBAN) can bring to welfare and healthcare fields, an interest relating to them has greatly increased recently. Wearable personal monitoring system enables, e.g., free movement and diverse monitoring of human vital signs automatically, without person's active action.

As in all wireless communications systems, good and sufficient knowledge of propagation channel characteristics is essential also in the WBAN system development work. With the channel information, a transceiver concept can be optimized to the final use-case in the best possible manner. Thus, WBAN radio channel modeling has been in a research agenda already for several years. Radio channel research is widely carried out in various research organizations all around the world and there is a large amount of published information already available. However, each varying installation, e.g., with respect to changes in antenna locations, frequency bands, surroundings conditions, or rate of mobility, has different influences on the propagating signals.

As defined in the original WBAN standard IEEE802.15.6 [1], ultra wideband (UWB) was selected as a mandatory technology for compliant wireless WBAN communications. Although the associated radio channel study group has released its own document describing the reference channel models [2] for system based on [1], there is still a need for further research and complementary channel models.

This has motivated also us at University of Oulu, Finland, to carry out research under this topic. So far, lots of radio channel related work has been done for on-body communications, thus where all the communicating sensor nodes are attached on the human body. Corresponding research where WBAN nodes are connected to off-body node, e.g., to room access point, have also been carried out. Both on-on and on-off-body channel studies and published works are based on extensive measurement campaigns.

Our latest WBAN related research activities have focused on communications where in-body signal propagation needs also to be taken into account [3]-[6]. Using advanced electromagnetic (EM) computing simulation tools, such as CST MicroWave Studio, it is possible to study how transmitted signal energy propagates inside a human body, underneath the skin and in the deeper tissues, such as gastro-intestine (GI) track. CST provides reasonable accurate voxel models for studies where the impact of human tissues is needed to be involved in the simulations. Because the in-body radio channel measurements using humans is problematic both ethically and from realization point of view, modern EM simulators provide a way and starting point for such a research. However, the open literature includes few in-body related channel studies, which are based on the experiments using, e.g., living pigs [7].

The movements of different parts of the human body affect also to the radio channel characteristics. Different postures and various limb or body movements are changing the "view" of an antenna to its communications link's counterpart. Thus, line-of-sight (LOS) link can every now and then be obstructed (OLOS) or even non-LOS (NLOS) as time goes by. The change in the channel characteristics in non-static cases has also been studied by experiments and reported, e.g., in [8]-[10].

\section{Studied Use Cases}

In this paper, two different WBAN communications approaches are discussed. Firstly, a summary of the findings from the EM computer simulations carried out between two on-body sensor nodes on a) chest and b) on abdomen area. Since the modern EM simulators could give 
rather accurate results for signal energy distribution in various complex environments, these tools are capable to simulate radio signal propagation also in the human body context. The CST's human voxel library includes several human voxel models, which have different body structures. Both genders are included as well. Especially beneficial for in-body propagation studies is that the voxel models contain almost all the inner organs, having their correct electromagnetic properties, as reported, e.g., in [11]-[12]. The latter reference provides an executable program, which can be used to calculate relative permittivity and conductivity values for different human tissues as a function of frequency.

When studying the impact of the tissues underneath the skin and deeper on the signal propagation on-body, our biggest interest has been in the chest and abdomen areas. According to our earlier studies, it was seen that metallic implants have an impact on the radio signal propagation characteristics [13]-[14]. Thus, more complementary measurements and simulations were carried out. The simulations were done using CST's four different human voxel models. In addition to the plain, reference simulations, artificial components illustrating aortic valve implant and/or sternotomy wires were embedded in the voxel models or layer models. E.g., sternotomy wires are used after an open surgery operation to close the sternum and thus, their possible impact needs to be considered. After time elapses, the wires are buried more deeply inside sternum. This has an impact also on their effect to on-body propagation. The implanted parts were modelled using their characteristic shapes and dielectric values to match reality as closely as possible.

The other use case of interest in this paper is focused on modeling dynamic on-off channel in the WBAN context. As already mentioned, the movement of a human body will cause significant changes in the channel characteristics, which has also straight impact on the WBAN system's performance. To understand the WBAN channel characteristics in a wider scope enables the system design in the best possible way. This part of the radio channel study was carried out by experiments.

The measurements were carried out using a vector network analyzer (VNA). The frequency bands covered ranges of 3$5 \mathrm{GHz}$ [8] and 2-8 GHz [9]-[10]. In [8], fast VNA sweeping time compared to the expected channel coherence time was used in order to enable data post-processing in timedomain. Large bandwidth enables high delay resolution, thus different multipath components can be distinguished accurately. In the case of dynamic measurements, narrower bandwidth is used. Pseudo-dynamic measurements can be carried out using the larger bandwidth because during the recordings, the movements were frozen, and body posture was changed between the measurements. Naturally, pseudo-dynamic measurements are not eligible to provide real statistics of the movement but provide insights how the channel could change. With respect to measurements on off-body cases, they can be performed in static, pseudo- dynamic or dynamic manner. With UWB signaling, fully dynamic measurement scenarios are challenging due to the question of channel coherence time demand, as reported in [8]. Measurement equipment often set practical limitations to the bandwidth examined, or the rate of dynamics to be observed. One clever way to circumvent these aspects, is to use pseudo-dynamic measurement approach.

\section{Results}

\subsection{For In-Body WBAN Communications}

In this chapter, we summarize the results of the impact of sternotomy wires on on-body propagation in abdomen area.

Due to the highly conductive features of metallic implants, they have impact also on the radio channel characteristics on-body. Closer the skin the conductive implants are, higher is their impact on on-body propagation. Metallic structures will create a propagation path, which allows radio signal to convey inside a body with smaller attenuation and higher velocity than if they are propagating through human tissues. In addition, metallic structures may have impact on the on-body antenna's performance by changing their ground and thus, the effect can be seen in changes of antennas' impedance matching, efficiency and radiation characteristics.

As presented in [3] and [15], using the elliptical layer model, the impact of the sternotomy wires on in-body propagation can be visualized. Figure 1a presents the power flow in the case wires are included in the model. The corresponding results without wires are shown in Figure $1 b$. The reference power flow level $(0 \mathrm{~dB})$ is set to the transmitting antenna. As seen, the signal reaches larger area when signal can propagate via wires. Complementary research is presented in [15], where the impact of aortic valve on channel characteristics is also studied.

a)

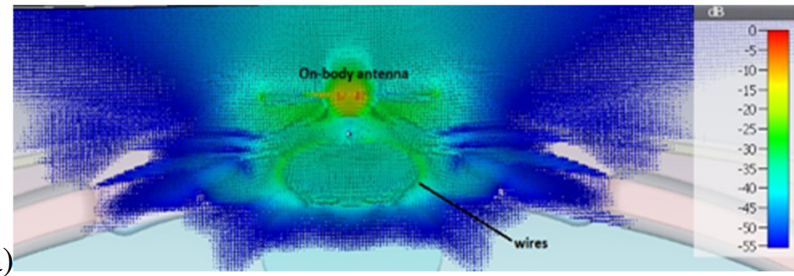

b)

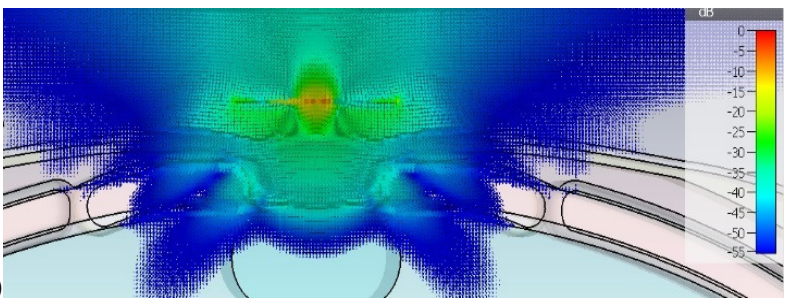

Figure 1. CST power flow results on the chest a) with and b) without sternotomy wires.

The simulation results also indicate that the UWB signal propagation in-body finds its way via tissues having lower 
relative permittivity $\left(\varepsilon_{\mathrm{r}}\right)$, and thus, smaller attenuation. Metallic implants and fat are the easiest media to propagate in-body. If comparing the relative permittivity values of fat and muscle at $4 \mathrm{GHz}, \varepsilon_{\mathrm{r}}=5.12$ and 50.82 [12], respectively, it is evident that the path loss is different through these tissues. Skin has also relatively high $\varepsilon_{\mathrm{r}}$, being 36.6 [12]. Thus, signal is assumed to propagate underneath the skin, through the fat layer, also in on-body links. The other propagation mechanism in on-body communications are reflections, scattering and creeping waves that circulate the body as a surface wave.

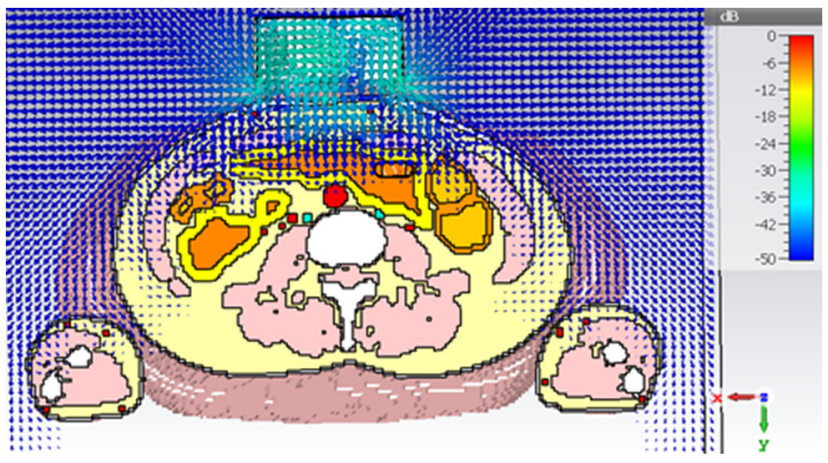

Figure 2. CST power flow results. Antenna location is on navel (top of the figure).

Similar propagation phenomena can be found when studying a communications link between on-body node and endoscope capsule. The transmitted signal is propagating via tissues having smaller relative permittivity. Thus, the received signal energy can be higher even in further capsule location corresponding to the closer distance if fat channel can be utilized. [5]

These effects discussed above are shown in Figure 2. The transmitted signal energy is favoring the tissues, which have smaller relative permittivity. On the other hand, where muscles are involved, the attenuation is high and penetration depth is small. Thus, it is important to take into account the tissues underneath the skin when locating the on-body antennas. [6]

\subsection{For On-Off-Body WBAN Communications}

This chapter is devoted to measurement-based studies on on-off -body channel. A 4-port VNA is used in the studies in such a way that one antenna locating in a pole is connected to VNA's port via cable. The other three ports are used to connect on-body antennas to the VNA. The recordings produced 16 S-parameter combinations, characterizing all the involved link combinations. Transmission coefficients were used to characterize the propagation channel as reflection coefficients indicated the goodness of the antenna matching.

A dynamic human movement is modeled in the measurements by conducting several static measurements, with several different human body postures, and then combining these different postures to model a dynamic motion, as in a cinema film. Figure 3 presents impulse response examples of such an approach with five body postures, describing a normal walking sequence, as originally presented in [10]. Figure 3 describes obtained channel impulse responses between dipole-typed antennas between the right wrist of a test person and a pole located two meters away the test person, at the height of two meters. In the measurements, the test person was facing towards the pole. As can be seen from Figure 3, the change in the body postures cause differences to the delays of the first arriving paths, as well as delay and power variations to other distinguished multipath components. Due to the measurement configuration, attenuation differences between the body postures are not large. It gives a result, that body shadowing difference effect in this specific measurement case is not large. Furthermore, the measurements were carried out at a frequency range 2-8 $\mathrm{GHz}$, in an anechoic chamber environment, in order to investigate body shadowing effect solely.

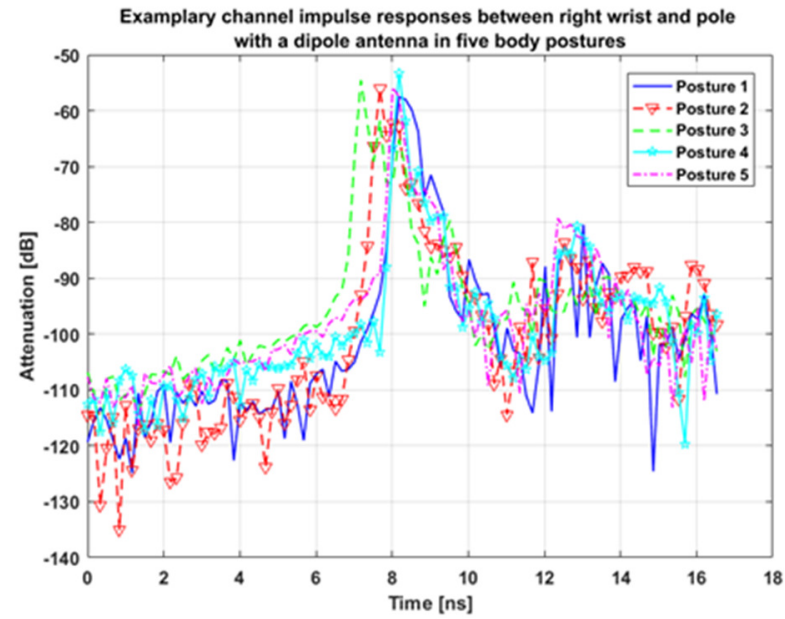

Figure 3. Exemplary impulse responses of an off-body pseudo-dynamic measurement scenario with five body postures.

As human dynamics has an impact on the signal propagation, it is also expected that the polarization is changed in the received signal. This effect has preliminary been studied and reported in [16].

\section{Conclusion}

The knowledge of the propagation characteristics inside a human body is mandatory when developing wireless communications systems for in-in-, in-on- and on-off-body communications. If during the on-body node installation the highly absorbing tissues underneath the skin/antenna can be avoided, it is possible to get signal propagating inbody penetrating longer. In medical applications, such as in capsule endoscopy, this could have significant impact on the system performance. Wireless body area networks operating in the close vicinity of a human body are favored to use as small transmission power as possible to retain radiation exposure safety limits. Thus, combining low transmission power and favorable in-body propagation 
channel could provide access to implant or endoscope capsules deeper inside a human body. Not only tissues, all the possible conductive implants should also be noted as conductive materials create simple paths for signal to propagate faster and longer.

As most of the WBAN nodes are still expected to locate onbody, it is essential to include information on radio channel characteristics also to on-on- and on-off-link studies. Body movement has significant impact on the received signal's statistics and thus, also the whole wireless transceiver chain's performance.

As a future work, more sensor locations should be studied for in-body use-cases. One application this channel information is valuable is localization of an endoscope capsule in GI track. There will also be need for implant to implant communications, which requires more research. In on-body part, especially dynamic body-to-body radio channel modeling needs more research. The WBAN channel modelling is still a vigorous research area.

\section{Acknowledgements}

This research has been financially supported in part by the self-funded project WBAN Communications in the Congested Environments (MeCCE), Academy of Finland 6Genesis Flagship (grant 318927) and the European Union's Horizon 2020 programme under the Marie Skłodowska-Curie grant agreement No. 872752.

\section{References}

[1] IEEE Computer Society, "IEEE Standard for local and metropolitan area networks-Part 15.6: Wireless Body Area Networks," IEEE Std 802.15.6 TM $^{\mathrm{T}} 2012$, $271 \mathrm{p}$.

[2] K. Yekeh Yazdandoost and K. Sayrafian-Pour, "Channel Model for Body Area Network (BAN)," doc: IEEE P802.15-08-0780-12-0006, 2010.

[3] M. Särestöniemi, C. Pomalaza-Ráez, Z. Bi, T. Kumpuniemi, C. Kissi, M. Sonkki, M. Hämäläinen, J. Iinatti, "Comprehensive Study on the Impact of Sternotomy Wires on UWB WBAN Channel Characteristics on the Human Chest Area," IEEE Access, Print ISSN: 2169-3536, Online ISSN: 21693536, DOI: 10.1109/ACCESS.2019.2920067.

[4] M. Särestöniemi, C. Pomalaza-Ráez, M. Berg, C. Kissi, M. Hämäläinen, J. Iinatti, "In-Body Power Distribution for Abdominal Monitoring and Implant Communications Systems," International Symposium on Wireless Communications Systems (ISWCS'2019), Oulu, Finland.

[5] M. Särestöniemi, C. Pomalaza-Ráez, M. Berg, C. Kissi, M. Hämäläinen, J. Iinatti, "UWB-WBAN Radio Channel Characteristics between the Endoscope Capsule and On-body Antenna Link," Bodynets 2019, Florence, Italy.

[6] M. Särestöniemi, C. Pomalaza-Ráez, C. Kissi, T. Kumpuniemi, M. Sonkki, M. Hämäläinen, J. Iinatti,
"Fat as a Propagation Medium in the Abdomen Area in the WBAN Applications," Bodynets 2019, Florence, Italy.

[7] C. Garcia-Pardo, A. Fornes-Leal, N. Cardona, R. Chávez-Santiago, J. Bergsland, I. Balasingham, S. Brovoll, Ø. Aardal, S.-E. Hamran, R. Palomar, "Experimental Ultra Wideband Path Loss Models for Implant Communications," 2016 IEEE 27th Annual IEEE International Symposium on Personal, Indoor and Mobile Radio Communications, Valencia, Spain.

[8] T. Kumpuniemi, J.-P. Mäkelä, M. Hämäläinen, K. Yekeh Yazdandoost, J. Iinatti, "Dynamic UWB OffBody Radio Channels - Human Body Shadowing Effect," the 28th Annual IEEE International Symposium on Personal, Indoor and Mobile Radio Communications, Montreal, Canada.

[9] T. Kumpuniemi, J.-P. Mäkelä, M. Hämäläinen, K. Yekeh Yazdandoost, J. Iinatti, "Measurements on Dynamic Off-Body Radio Channels at UWB Frequencies," 2019 13th International Symposium on Medical Information and Communication Technology, Oslo, Norway.

[10] T. Kumpuniemi, J.-P. Mäkelä, M. Hämäläinen, J. Iinatti, "Pseudo-Dynamic UWB WBAN Off-Body Radio Channel Measurements - Preliminary Results," Bodynets 2019, Florence, Italy.

[11] C. Gabriel, "Compilation of the Dielectric Properties of Body Tissues at RF and Microwave Frequencies," AFOSR-TR-96, 1995.

[12] http://niremf.ifac.cnr.it/tissprop/

[13] M. Hämäläinen, A. Taparugssanagorn, R. Tesi, J. Iinatti, "Wireless Medical Communications Using UWB," 2009 IEEE International Conference on Ultra Wideband, Vancouver, Canada, 2009.

[14] W.-B. Yang, K. Sayrafian-Pour, J. Hagedorn, J. Terrill, K. Y. Yazdandoost, A. Taparugssanagorn, M. Hämäläinen, J. Iinatti, "Impact of an Aortic Valve Implant on Body Surface UWB Propagation: A Preliminary Study," the $5^{\text {th }}$ International Symposium on Medical Information and Communication Technology, Montreux, Switzerland, 2011.

[15] M. Särestöniemi, C. Pomalaza-Raez, T. Kumpuniemi, M. Hämäläinen, R. Kovacs, J. Iinatti, ”Measurement Data-Based Study on the Intrabody Propagation in the Presence of the Sternotomy Wires and Aortic Valve Implant," IEEE TAP, Vol. 67, Iss. 8, 2019.

[16] K. Turbic, M. Särestöniemi, M. Hämäläinen, T. Kumpuniemi, L.M. Correia, ”A Preliminary Analysis of User's Body Impact on Signal Polarization in WBANs," the $14^{\text {th }}$ European Conference on Antennas and Propagation, Copenhagen, Denmark, 2020. 